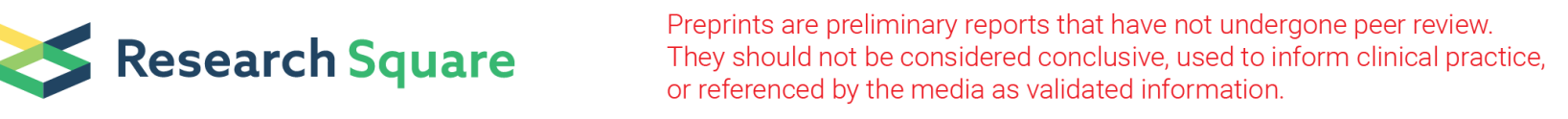

\title{
Transcriptional regulatory networks mediated by abnormal m6A modifications in tumor development
}

Wenzheng Qu ( $\square$ quwz@zju.edu.cn )

Zhejiang University School of Medicine Children's Hospital https://orcid.org/0000-0002-1150-295X

Xuekun Li

Zhejiang University School of Medicine

Research

Keywords: RNA methylation, N6-methyladenosine, gene expression, transcriptional regulatory, tumor development

Posted Date: May 17th, 2021

DOI: https://doi.org/10.21203/rs.3.rs-523230/v1

License: (1) This work is licensed under a Creative Commons Attribution 4.0 International License. Read Full License 


\section{Abstract \\ Background}

N6-methyladenosine $\left(\mathrm{m}^{6} \mathrm{~A}\right)$ is one of the common modifications of transcripts that is regulated by related proteins including writers, readers and erasers. Abnormal $\mathrm{m}^{6} \mathrm{~A}$ modification plays an important role in the process of tumor development. Current approaches for tumor $\mathrm{m}^{6} \mathrm{~A}$ RNA methylation, based on sequencing data from public databases, focus on the comparison of $\mathrm{m}^{6} \mathrm{~A}$ modification regulator expression by RNA-seq data.

\section{Results}

We obtained MeRIP-seq and corresponding RNA-seq data from GEO database to compare and analyze the distributional characteristics of $\mathrm{m}^{6} \mathrm{~A}$ in different types of tumor samples, the differences in $\mathrm{m}^{6} \mathrm{~A}$ modification enrichment, and the regulatory role in gene transcriptional expression level. We found that the enrichment of $\mathrm{m}^{6} \mathrm{~A}$ modification was enhanced in IMPA and GBM tumor samples, but was decreased in METTL3 knockdown tumor cells. Combined with clinical data from the TCGA database, we found that the high expression of DUSP7 significantly affected the overall survival of AML patients and was involved in tumor development through the PIK3R2 protein and MAPK signaling pathways.

\section{Conclusion}

Abnormal $\mathrm{m}^{6} \mathrm{~A}$ modification enrichment in tumor samples leads to dysregulation in the expression of cancer-related genes.

\section{Background}

Post-transcriptional modification has become an important regulator in many biological processes and has attracted increasing attention. N6-methyladenosine $\left(m^{6} A\right)$ is one of the common internal modifications in eukaryotic mRNA[1]. One of the methods widely used to study $\mathrm{m}^{6} \mathrm{~A}$ modification at the transcriptional level is methylated RNA immunoprecipitation sequencing (MeRIP-seq), which involves the immunoprecipitation of $\mathrm{m}^{6} \mathrm{~A}$ modified RNA fragment and peak detection by comparing to background gene coverage. In 2012, Meyer et al. [2] determined 7,676 human genes containing $\mathrm{m}^{6} \mathrm{~A}$ in somatic cells using the MeRIP-seq method; they found that $\mathrm{m}^{6} \mathrm{~A}$ was primarily localized to the conserved RRACH sequence $(R=G$ or $A ; H=A, C$ or $U)$, the sequence is enriched in the $3^{\prime}$ untranslated regions( $\left.3^{\prime} U T R s\right)$, and the stop codon region of the protein-coding mRNA. In mammals the amount of $\mathrm{m}^{6} \mathrm{~A}$ modified adenine is between $0.1 \%$ and $0.4 \%[3,4]$, so each mRNA has only $3-5 \mathrm{~m}^{6} \mathrm{~A}$ methylation sites and regulates RNA stability, localization, splicing, transport, and translation at the post-transcriptional level[5]. 
$\mathrm{m}^{6} \mathrm{~A}$ modification is mainly associated with three types of regulators. The first is $\mathrm{m}^{6} \mathrm{~A}$ methyltransferases, which encode genes called "writers" and include METTL3, MetTL14, MetTL16, RBM15/15B, KIAA1429, ZC3H13 and WATP (Wilms Tumor 1 associated protein)[6]. Together, they form a complex that induces the writing of the $\mathrm{m}^{6} \mathrm{~A}$ methylated group into RNA [7]. The second type of regulators is $\mathrm{m}^{6} \mathrm{~A}$ demethylase, which encodes genes called "erasers" and includes FTO and ALKBH5 [8]. This protein can remove the $\mathrm{m}^{6} \mathrm{~A}$ methylation group in RNA, thus affecting the biological process of tumor. The last group of regulators can bind to the $\mathrm{m}^{6} \mathrm{~A}$ methylation site and can read information to play a role; the encoding genes of this group are called "readers", some of which identified so far are YTHDF1, YTHDF2, YTHDF3,YTHDC1, YTHDC2, HNRNPC, HNRNPG, HNRNPA2B1 and IGF2BPs[9]. Readers are proteins that bind to the $\mathrm{m}^{6} \mathrm{~A}$ sites on RNA in the nucleus or cytoplasm to perform a specific biological function. At present, the research on the molecular biological mechanism of $\mathrm{m}^{6} \mathrm{~A}$ modification of tumor is still in the initial stage, and further exploration is needed to provide targets for tumor therapy.

Breakthroughs in the discovery and research of $\mathrm{m}^{6} \mathrm{~A}$ writers, erasers and readers, as well as the development of high-throughput sequencing data analysis, have helped illuminate the biological functions and potential mechanisms of $m^{6} \mathrm{~A}$. A growing number of studies have shown that $\mathrm{m}^{6} \mathrm{~A}$ RNA methylation plays an important role in tumor genesis and development. The effect of $\mathrm{m}^{6} \mathrm{~A}$ modification on tumors is mainly reflected in the regulation of these tumor-related genes. Abnormal $\mathrm{m}^{6} \mathrm{~A}$ modifications are closely related to tumor development $[10,11]$. Therefore, it is particularly important to further study the regulatory mechanism of $\mathrm{m}^{6} \mathrm{~A}$ modification in the process of tumor development. Glioblastoma is the most deadly primary brain tumor. Studies have shown that $\mathrm{m}^{6} \mathrm{~A}$ modification is associated with the growth and self-renewal of glioblastoma stem cells (GSCs)[12]. Knockdown of METTL3 or METTL14, key components of the RNA methyltransferase complex, promotes GSCs growth, self-renewal, and tumorigenesis, whereas upregulation of METTL3 or inhibition of FTO has the reverse effects[12]. Another study also reported the important role of METTL3-mediated $\mathrm{m}^{6} \mathrm{~A}$ modification in GSC maintenance and in glial cell dedifferentiation[13]. This study found that METTL3 expression is upregulated in GSCs and is attenuated during differentiation. The role of METTL3 has also been reported in acute myeloid leukemia(AML). Studies have shown that METTL3 is expressed more abundantly in AML cells than in normal hematopoietic cells. And METTL3-mediated elevation of $\mathrm{m}^{6} \mathrm{~A}$ in AML plays an important role in maintaining pluripotency and in inhibiting cell differentiation $[14,15]$. In other tumors, such as gastric cancer, upregulation of METTL3 promotes the stability of ZMYM1, thereby enhancing EMT process in vitro and metastasis in vivo[16].

In the present study we obtained high-throughput sequencing data of MeRIP-seq for invasive malignant pleomorphic adenoma (IMPA), glioblastoma (GBM), acute myeloid leukemia(AML), and gastric cancer (GC) from GEO database, and we analyzed the level of $\mathrm{m}^{6} \mathrm{~A}$ modification in different tumor samples. Combined with the corresponding RNA sequencing (RNA-seq) data of these tumor samples, we analyzed the differences of gene expression at the transcription level under different $\mathrm{m}^{6} \mathrm{~A}$ modification levels. In addition, combined with the tumor clinical data from the The Cancer Genome Atlas (TCGA) RNA-seq 
database, we expected to identify the key genes that affect the survival of different tumor patients under the regulation of $\mathrm{m}^{6} \mathrm{~A}$ modification, so as to provide guiding strategies for the clinical treatment of tumor patients. Based on this data analysis, we found that the enrichment degree of $\mathrm{m}^{6} \mathrm{~A}$ modification was enhanced in IMPA and GBM but was decreased in METLL3 or YTHDF1 knockdown tumor cell lines, suggesting that $\mathrm{m}^{6} \mathrm{~A}$ modification plays an important role in tumorigenesis. Among the genes with different levels of $\mathrm{m}^{6} \mathrm{~A}$ modification that were common to different tumor types, the differential expression of DUSP7 is significantly correlated with the survival of patients with AML. DUSP7 may be involved in tumor genesis and development through the MAPK signaling pathway, and this gene has rarely been reported in AML, thus providing a new idea for subsequent clinical studies on AML.

\section{Results}

\section{Peak Identification and Distribution Pattern of $\mathrm{m}^{6} \mathrm{~A}$ Modification Enrichment in Different Types of Tumor Samples}

We screened MeRIP-seq data for different tumor types from the GEO database, but there was less data related to tumor tissue samples. After screening, we obtained MeRIP-seq and RNA-seq data of Invasive Malignant Pleomorphic Adenoma (IMPA), Glioblastoma (GBM), Acute myeloid leukemia (AML), and Gastric Cancer (GC) from the GEO database (Figs. 1A). The IMPA data (GSE161879) were derived from five pairs of tumor tissue samples from invasive malignant pleomorphic adenoma tumor tissue specimens and corresponding normal glandular tissue. GBM data (GSE158741) were derived from four pairs of neural stem cells and glioblastoma stem cells derived from glioblastoma patients. AML data (GSE94613) were derived from METTL3 knockdown MOLM13 cells (4 samples) and control cells (2 samples). GC data (GSE166972) were obtained from 3 pairs of YTHDF1 knockdown AGS cell samples and control cells.

First, we identified the enrichment of peaks for these tumor samples. Then the sample correlation analysis is carried out based on the enrichment degree of different samples of peaks. Clustering analysis was performed for IMPA samples according to Pearson correlation, and 4 outliers were removed according to the clustering results (Figures S1A). Hierarchical cluster analysis was performed for other types of tumors. Based on the GBM peak-calling results, the two samples (GSC_rep2 and GSC_rep4) identified the least peaks and affected the clustering of samples within the group, so they were excluded in the subsequent analysis (Figures S1B). The sample data of AML and GC were not deleted (Figures S1C-D). Then peaks within the group were merged, and the distribution of these peaks was analyzed in $5^{\prime}$ untranslated regions (5' UTR), coding sequences (CDS), 3' untranslated regions ( $3^{\prime}$ UTR), TSS (defined from TSS $-1 \mathrm{~kb}$ to $+100 \mathrm{bp}$ ) and TTS (defined from TTS $-100 \mathrm{bp}$ to $+1 \mathrm{~kb}$ )[17].

After AML and GC tumor cells knockdown METLL3 and YTHDF1, respectively, the proportion of the number of peaks in the transcript region decreases (Fig. 1B). In IMPA tumor samples, there is no 
significant change in the proportion of the number of peaks (Figs. 1C). In glioblastoma stem cells, the proportion of the number of peaks in different regions decreases (Figs. 1D). According to metagene analysis of the $\mathrm{m}^{6} \mathrm{~A}$ enrichment peaks, we found that the $\mathrm{m}^{6} \mathrm{~A}$ peaks of GBM are enriched in $5^{\prime}$ UTR, $3^{\prime}$ UTR and near the stop codons (Figs. $1 \mathrm{H}$ ), whereas other tumor samples are enriched in 3' UTR and near the stop codons (Figs. 1E-G).

\section{The Increased Enrichment Level of $\mathrm{m}^{6} \mathrm{~A}$ Peaks in IMPA and GBM and Decreased Enrichment in AML METLL3 Knockdown}

The $\mathrm{m}^{6} \mathrm{~A}$ peaks in these tumor samples were characterized by the canonical GGAC motif (Figs. 2A, Figures S2A). The threshold value of difference peak is defined as the absolute value of foldchange greater than 2 and a p-value less than 0.05. The differential enrichment peaks of IMPA included 1772 hyper-methylated $\mathrm{m}^{6} \mathrm{~A}$ peaks and 1156 hypo-methylated $\mathrm{m} 6 \mathrm{~A}$ peaks. AML included 805 hyper-methylated $\mathrm{m}^{6} \mathrm{~A}$ peaks and 1480 hypo-methylated $\mathrm{m} 6 \mathrm{~A}$ peaks. GC included 560 hyper-methylated $\mathrm{m}^{6} \mathrm{~A}$ peaks and 710 hypo-methylated $\mathrm{m}^{6} \mathrm{~A}$ peaks. GBM included 3321 hyper-methylated $\mathrm{m}^{6} \mathrm{~A}$ peaks and 1939 hypomethylated $\mathrm{m}^{6} \mathrm{~A}$ peaks (Figs. $2 \mathrm{~B}$ ). According to the accumulation distribution analysis of group peaks enrichment value, we found that the enrichment level of $\mathrm{m}^{6} \mathrm{~A}$ peak was increased in IMPA and GBM compared with the control group (p-value < E-10) (Figs. 2C-D), but was decreased in AML cells after METTL3 knockdown (p-value = 8.37E-16) (Figs. 2E). After YTHDF1 knockdown in GC cells, the enrichment level did not change significantly (Figs. 2B).

Functional enrichment analysis was performed based on the screened differential peak associated genes. In IMPA, compared with the hypo-methylated peaks, the hyper-methylated peak associated genes were enriched in more cancer-related signaling pathways ( $p$-value $<0.05$ ), such as p53 signaling pathway, Hedgehog signaling pathway, PI3K-Akt signaling pathway, etc. (Fig. 2F). In GBM, both the hypomethylated peaks and hyper-methylated peak associated genes were enriched in some cancer-related pathways, such as PI3K-Akt signaling pathway, p53 signaling pathway, WNT signaling pathway, HIF-1 signaling pathway, and TNF signaling pathway, etc. (Fig. 2G). After knocking down METLL3 in AML cells, compared to the control, hypo-methylated peak associated genes enrichment in more cancer related pathways (Fig. 2H). However, after YTHDF1 knockdown in GC cells, there was no significant difference in the enrichment results of cancer-related pathways between genes associated with hyper-methylated and hypo-methylated peaks (Fig. 2H).

\section{Associations Between $\mathrm{m}^{6} \mathrm{~A}$ Enrichment Peaks with Dysregulation and Differentially Expressed Genes in Tumors}

To investigate the association between differential enrichment peaks and gene expression, we further analyzed RNA-seq data from these tumor samples. The threshold value of differentially expressed genes was defined as the absolute value of foldchange greater than 2 and a $p$-value less than 0.05 . The IMPA tumor group consisted of 1778 up-regulated genes and 1459 down-regulated genes compared with the control group. Compared with the control, GSC included 2554 upregulated and 2253 downregulated 
genes. After METTL3 knockdown, AML cells included 1555 upregulated and 1089 downregulated genes, whereas GC cells included 830 upregulated and 387 downregulated genes after YTHDF1 knockdown (Fig. 3A).

Differential $\mathrm{m}^{6} \mathrm{~A}$ methylation peaks associated genes were analyzed combined with differential expression genes (Fig. 3B), and we found that in IMPA tumor and GSC group, there were more genes that significantly upregulated with hyper-methylated $\mathrm{m}^{6} \mathrm{~A}$ peaks (hyper-up) and significantly downregulated with hypo-methylated $\mathrm{m}^{6} \mathrm{~A}$ peaks (hypo-down). To compare the changes in expression of $\mathrm{m}^{6} \mathrm{~A}$-modified and unmodified genes, cumulative distribution analysis was performed based on foldchange of gene expression[18]. A significant influence of $\mathrm{m}^{6} \mathrm{~A}$ modification on gene expression was found in IMPA and GBM group (p-value< E-06) but not in AML and GC knockdown group (Fig. 3C-D).

Further functional enrichment analysis was performed on the differentially expressed genes associated with differential enrichment peaks. It was found that in the IMPA tumor group, the differentially expressed genes associated with hyper-methylated peaks were enriched in more cancer-related pathways ( $p$-value < 0.05) than genes associated with hypo-methylated peaks (Fig. 3E). In the GSC group, compared with the hyper-methylated peaks, the different regulated genes associated with the hypo-methylated peaks were enriched in more cancer-related pathways (p-value < 0.05) (Fig. 3F). In AML and GC knockdown cells, differentially $\mathrm{m}^{6} \mathrm{~A}$ enrichment peaks associated with different regulated genes were enriched in fewer cancer-related pathways than IMPA and GBM group (p-value < 0.05) (Fig. 3G-H). However, GO analysis for AML knockdown cells group showed that the differentially expressed genes that hypo-methylated were related to cell differentiation (Supplementary Table 1).

\section{Specificity of Differentially Expressed Genes Associated with Clinical Overall Survival Mediated by $\mathrm{m}^{6} \mathrm{~A}$ Modifications in Tumors}

To explore whether common $\mathrm{m}^{6} \mathrm{~A}$ modification-mediated differentially expressed genes (DEGs) in different tumors are associated with overall survival in patients, we analyzed the intersection of these gene sets. The GC group was not considered in the common analysis because the enrichment in cancerrelated pathways was not significant. There were 140 DEGs and 185 differentially $\mathrm{m}^{6} \mathrm{~A}$ methylation associated genes (DMGs) in IMPA, GBM, and AML (Fig. 4A). And there were 10 genes within both DEGs and DMGs (Fig. 4B). The expression patterns of these 10 genes in the three tumor groups were not completely consistent (Fig. 4C).

Combined with clinical data from the TCGA RNA-seq database, we analyzed the overall survival of these 10 genes in different tumor patients. Because there are no clinical data of IMPA in the TCGA database, the data of Head and Neck Squamous Cell Carcinoma (HNSC) were selected for analysis. The differential expression of DUSP7 ( $p$-value $=0.00051$ ) was significantly correlated with overall survival in AML patients $(n=173)(p$-value $=0.0025)(F i g .4 D)$. High expression of DUSP7 was associated with poor survival in AML patients. DUSP7 is a hypo-methylated associated gene in AML sh-METLL3 cells, containing two hypo-methylated peaks (chr3:52050843-52051063, p-value $=0.00173$; chr3:52053994- 
$52054343, p$-value $=0.00515)$ (Figure S2C), which is consistent with the visualization result of the peaks on DUSP7 (Fig. 4E). High expression of other genes, such as IL31RA ( $p$-value $=0.18$ ), was associated with poor overall survival in HNSC patients $(n=566)(p$-value $=0.044)(F i g .4 F)$, the high expression of COL6A1 ( $p$-value $=0.24)$ and $\cos 2(p$-value $=0.69)$ was associated with poor overall survival of GBM patients $(n=$ 166) $(p$-value = 0.01) (Fig. 4D); however, differences in the expression of these genes were not significant. For the gene set common to DEGs and DMGs in GC, no genes were found to be significantly associated with the overall survival of patients with stomach adenocarcinoma (STAD) $(n=41)$ (Figure S2D).

\section{DUSP7 Involved in the Development of AML Through PIK3R2 Protein and MAPK Signaling Pathways}

To further investigate the effect of high expression of DUSP7 on poor survival in AML patients, the RNAseq cohort data $(n=173)$ from TCGA databases were divided into a high expression group and a low expression group based on the median expression value of DUSP7. Based on the expression value of DUSP7 in the two groups, 19,894 genes were ranked in the TOP10 (DUSP7 ${ }^{\text {High }}$ ) vs. bottom10 (DUSP7 ${ }^{\text {Low })}$ for gene set enrichment analyses (GSEA) $(n=20)$. It was found that the DUSP7 ${ }^{\text {High }}$ tumor samples were enriched in chemokine signaling pathway, JAK-STAT signaling pathway and MAPK signaling pathway ( $p$ value < 0.05) (Fig. 5A) compared with the DUSP ${ }^{\text {Low }}$ group.

In addition, based on the expression value of DUSP7 in samples, GSEA analysis was carried out according to the ranking of person correlation in the DUSP7 positively correlated (DUSP7Pos) vs. DUSP7 negatively correlated (DUSP7 ${ }^{\mathrm{Neg}}$ ) [19]. Compared with the DUSP7 ${ }^{\mathrm{Neg}}$ group, the DUSP7 ${ }^{\mathrm{Pos}}$ group was enriched in apoptosis signaling pathway, MAPK signaling pathway, and acute myeloid leukemia pathway (p-value < 0.05) (Fig. 5B). DUSP7 belongs to a class of DUSPs that inactivates MAPK through dephosphorylation [20]. Because both GSEA results are enriched in the MAPK signaling pathway, to further analyze the interaction pathway associated with DUSP7 in this pathway, the protein interaction analysis based on STRING database was performed. DUSP7 was found to interact with MAPK1, MAPK3, and PIK3R2 (Fig. 5C). PIK3R2, a phosphoinositide-3-kinase regulatory subunit 2, is involved in some cancer-related pathways , such as the JAK-STAT signaling pathway, which was found to be enriched in all enrichment results of both GSEAs ( $p$-value < 0.05$)$.

\section{Discussion}

The occurrence and development of tumors are regulated by complex biological processes. As one of the modification types of transcript level, $\mathrm{m}^{6} \mathrm{~A}$ modification plays an important role in the metabolism of transcript [21-23]. More and more studies have shown that $\mathrm{m}^{6} \mathrm{~A}$ modification plays an important role in the process of tumor genesis and development $[16,24,25]$. To explore the biological functions of $\mathrm{m}^{6} \mathrm{~A}$ modification in different tumors, we obtained MeRIP-seq and RNA-seq data from GEO database for four tumor types to compare the differences in the level of $\mathrm{m}^{6} \mathrm{~A}$ modification and the biological signaling pathways involved in different tumor types. Combined with TCGA database, the effect of differentially 
expressed genes with significantly different $\mathrm{m}^{6} \mathrm{~A}$ modification on the overall survival of patients was analyzed.

MeRIP-seq analysis showed that $\mathrm{m}^{6} \mathrm{~A}$ modification was enriched in 3 'UTR, CDS, and $5^{\prime}$ UTR in different tumor samples. In tumor cells that knock down METLL3 or YTHDF1, the number of $m^{6} A$-enriched peaks on the transcript is reduced. METTL3 is an important $\mathrm{m}^{6} \mathrm{~A}$ RNA methylation transferase, and YTHDF1 is one of the $\mathrm{m}^{6} \mathrm{~A}$ readers[9]. Their expression in knockdown cells is reduced, which will affect the $\mathrm{m}^{6} \mathrm{~A}$ modification of the transcript. The cumulative distribution of peak enrichment showed that the overall $\mathrm{m}^{6} \mathrm{~A}$ enrichment level decreased significantly after AML cells knockdown METLL3. In IMPA and GBM tumor samples without gene knockdown, the overall enrichment level of $\mathrm{m}^{6} \mathrm{~A}$ was significantly increased. KEGG pathway enrichment results of the genes associated with different $\mathrm{m}^{6} \mathrm{~A}$ enrichment peaks indicated that the hyper-methylated peaks were significantly enriched in more cancer-related pathways in IMPA, while both the hyper-methylated and hypo-methylated peaks in GBM were enriched in more cancer pathways. These results suggest that abnormal $\mathrm{m}^{6} \mathrm{~A}$ modification of the transcript is involved in tumor progression.

In order to analyze the regulation of $\mathrm{m}^{6} \mathrm{~A}$ modification on transcription expression, combined with RNAseq data, the overall foldchange level of expressed genes were compared. The results showed that genes with $\mathrm{m}^{6} \mathrm{~A}$ modification (Gene ${ }^{\mathrm{m} 6 \mathrm{~A}}$ ) were more differentially expressed in IMPA and GBM tumor samples than those without $\mathrm{m}^{6} \mathrm{~A}$ modification (Gene $\mathrm{P}^{\mathrm{no}-\mathrm{m} 6 \mathrm{~A}}$ ), which suggests that the differential $\mathrm{m}^{6} \mathrm{~A}$ modification in tumors has a significant effect on the expression of transcripts. There was no significant change between Gene ${ }^{\mathrm{m} 6 \mathrm{~A}}$ and Gene $e^{\text {no-m6A }}$ in the tumor cells that knocked down METTL3 or YTHDF1 due to the decrease of the transcription level of $\mathrm{m}^{6} \mathrm{~A}$ modification. KEGG enrichment analysis of hyper-methylated DEGs (hyper-DEGs) and hypo-methylated DEGs (hypo-DEGs) indicated that these differentially expressed genes regulated by abnormal $\mathrm{m}^{6} \mathrm{~A}$ modifications were involved in cancer-related signaling pathways. However, in IMPA tumor samples, hyper-DEGs were involved in more cancer signaling pathways, while in GBM tumor samples, hypo-DEGs were involved in more cancer-related pathways, suggesting that the regulation of gene expression mediated by abnormal modifications of $\mathrm{m}^{6} \mathrm{~A}$ is a very complex regulatory network and that other regulatory factors may be involved.

Based on MeRIP-seq data, it was found that METTL3 knockdown resulted in the enrichment of hypomethylated peaks in the CDS region of DUSP7 transcripts, and the expression of this gene was significantly decreased. Combined with the TCGA RNA-seq database, we found that DUSP7 gene expression was significantly different in AML patients and that patients with high expression of DUSP7 gene had a shorter overall survival. Because there are few studies on DUSP7 in AML patients, further GSEA analysis of DUSP7 showed that it participates in MAPK signaling pathway through the MAPK1 and MAPK3, and it participates in the JAK-STAT signaling pathway through the PIK3R2 protein.

Phosphoinositol 3-kinase (PI3K) and mitogen-activated protein kinase (MAPK) have been widely reported for their roles in oncogenic transformation, cell cycle and apoptosis regulation, which are well-known cancer markers[26]. 
In summary, our article reveals the differences of $\mathrm{m}^{6} \mathrm{~A}$ modification enrichment in different types of tumors and shows that abnormal $\mathrm{m}^{6} \mathrm{~A}$ modification enrichment in tumor samples leads to differences in the expression of cancer-related genes. DUSP7 is significantly differentially expressed in AML patients, and its low expression is regulated by abnormal modification of $\mathrm{m}^{6} \mathrm{~A}$, while its high expression is associated with poor overall survival. DUSP7 has rarely been reported in AML, so it provides a new target for clinical study of AML.

\section{Conclusions}

$\mathrm{m}^{6} \mathrm{~A}$ modification was enriched in IMPA and GBM tumor samples but was decreased in METTL3 knockdown tumor cells. The high expression of DUSP7 significantly affected the overall survival of AML patients and was involved in tumor development through the PIK3R2 protein and MAPK signaling pathways.

\section{Methods}

\section{Sequencing Datasets and Clinical Data}

MeRIP-seq and RNA-seq data were obtained from the GEO database for different tumor types, including invasive malignant pleomorphic adenoma, glioblastoma, acute myeloid leukemia and gastric cancer. The sequencing data of invasive malignant pleomorphic adenoma were obtained from GSE161879, including 5 pairs of tumor tissues and corresponding normal gland tissues. The sequencing data of glioblastoma were obtained from GSE158741, including 4 pairs of neural stem cells and glioblastoma stem cells isolated from the patient's tumor tissue. The sequencing data of acute myeloid leukemia were obtained from GSE94613, including four METTL3 knockdown AML cell samples and two control samples. The sequencing data of gastric cancer were obtained from GSE166972, including 3 pairs of YTHDF1 knockdown AGS cell samples and control samples. Clinical data for tumors were obtained from The Cancer Genome Atlas (TCGA) RNA-seq database.

\section{Data Analysis for MeRIP-seq}

Raw reads of each sample were trimmed using the Trimmomatic software to remove adaptor sequences and bases with low quality [27]. The processed reads were then aligned to the human reference genome (version hg38, UCSC) using bowtie2 [28] with default parameters. Only unique mapped reads with mapping quality no less than 30 were kept for the subsequent analysis.

For MeRIP-seq peak calling, the MACS2 software was used to identity m6A-enriched (version 2.2.7) [29], with the corresponding input sample serving as control. MACS2 was run with default options except for'-p 0.05 -nomodel, -keepdup all'to turn off fragment size estimation and to keep all uniquely mapping reads, respectively. For differential peak analysis, DiffBind [30] packages was used to identify differentially enrichment peaks. The differentially peaks were selected with log2 (fold change) $>1$ or log2 (fold change) 
$<-1$ and $p$ value $<0.05$. Motifs enriched in m6A peaks within all mRNAs were identified using HOMER software (v4.10) [31]. The motif length was restricted to 5-6 nucleotides.

\section{RNA-Seq Analysis}

For gastric cancer pair-end sequencing data, Hisat2 [32] was used to map reads to the genome of Homo sapiens (hg38) with default parameters. For other tumor RNA-seq data, bowtie2 [28] was used to align reads to the human reference genome. featureCounts 1.5.0-p3 was used to count the reads numbers mapped to each gene. And FPKM of each gene was calculated based on the length of the gene and reads count mapped to this gene. The differential expression genes between samples were analysed with DESeq2. P-value of 0.05 and absolute foldchange of 2 were set as the threshold for significantly differential expression.

\section{Functional Enrichment Analysis}

Gene Ontology (GO) enrichment analysis of the differentially expressed genes and differentially enrichment peak related genes was implemented by clusterProfiler R package. GO terms with corrected Pvalue less than 0.05 were considered significantly enriched by differential expressed genes. And then test the statistical enrichment of differential expression genes in KEGG pathways. The gene interaction network was constructed by the STRING protein interactome database [33].

\section{Gene Set Enrichment Analysis (GSEA)}

To explore DUSP7 gene involving pathways that detected from RNA-seq data of LAML patients which obtained from TCGA, gene set enrichment analysis (GSEA) was performed with the JAVA program using the KEGG pathway gene set access from MSigDB. Genes were ranked on the basis of signal2noise between the high-expression and low-expression groups. GSEA analysis was also carried out according to the ranking of person correlation in the DUSP7 positively correlated vs. DUSP7 negatively correlated. Gene sets with P-value $<0.05$ after performing a GSEA preranked analysis were considered significantly enriched.

\section{Statistical Analysis}

Computational and statistical analyses were performed using R (version 4.0.4). Kaplan-Meier analysis and a log-rank test were used to conduct survival analysis. The Wilcoxon signed-rank test was applied to compare cumulative distribution. Statistical significance was defined as a p-value $<0.05$.

\section{Declarations}

\section{Ethics approval and consent to participate}

Not necessary.

\section{Consent for publication}


Not applicable.

\section{Competing interests}

The authors declare that they have no competing interests.

\section{Availability of data and materials}

Publicly available datasets were analyzed in this study. The MeRIP-seq data and RNA-seq data can be found from GEO database(https://www.ncbi.nlm.nih.gov/geo).

\section{Authors' contributions}

X.L. conceived and designed the project. W.Q. collected the clinical information, MeRIP-seq data, RNA-seq data and analyzed data. W.Q. and X.L. wrote the manuscript. All authors contributed to the article and approved the submitted version.

\section{Funding}

This study was supported by the National Key Research and Development Program of China (No. 2016YFC0900400).

\section{Acknowledgements}

Not applicable.

\section{References}

1. Yue Y, Liu J, He C. RNA N6-methyladenosine methylation in post-transcriptional gene expression regulation. Genes Dev. 2015;29(13):1343-55.

2. Meyer KD, Saletore $Y$, Zumbo P, Elemento $O$, Mason CE, Jaffrey SR. Comprehensive analysis of mRNA methylation reveals enrichment in 3' UTRs and near stop codons. Cell. 2012;149(7):1635-46.

3. Desrosiers R, Friderici K, Rottman F. Identification of methylated nucleosides in messenger RNA from Novikoff hepatoma cells. Proc Natl Acad Sci U S A. 1974;71(10):3971-5.

4. Wei CM, Gershowitz A, Moss B. Methylated nucleotides block $5^{\prime}$ terminus of HeLa cell messenger RNA. Cell. 1975;4(4):379-86.

5. Fu Y, Dominissini D, Rechavi G, He C. Gene expression regulation mediated through reversible m(6)A RNA methylation. Nat Rev Genet. 2014;15(5):293-306.

6. Roundtree IA, Evans ME, Pan T, He C. Dynamic RNA Modifications in Gene Expression Regulation. Cell. 2017;169(7):1187-200.

7. Schwartz S, Mumbach MR, Jovanovic M, Wang T, Maciag K, Bushkin GG, et al. Perturbation of m6A writers reveals two distinct classes of mRNA methylation at internal and 5 ' sites. Cell Rep. 
2014;8(1):284-96.

8. Huang Y, Yan J, Li Q, Li J, Gong S, Zhou H, et al. Meclofenamic acid selectively inhibits FTO demethylation of m6A over ALKBH5. Nucleic Acids Res. 2015;43(1):373-84.

9. Meyer KD, Jaffrey SR. Rethinking m(6)A Readers, Writers, and Erasers. Annu Rev Cell Dev Biol. 2017;33:319-42.

10. Pan Y, Ma P, Liu Y, Li W, Shu Y. Multiple functions of m(6)A RNA methylation in cancer. J Hematol Oncol. 2018;11(1):48.

11. Dai D, Wang H, Zhu L, Jin H, Wang X. N6-methyladenosine links RNA metabolism to cancer progression. Cell Death Dis. 2018;9(2):124.

12. Cui Q, Shi H, Ye P, Li L, Qu Q, Sun G, et al. m(6)A RNA Methylation Regulates the Self-Renewal and Tumorigenesis of Glioblastoma Stem Cells. Cell Rep. 2017;18(11):2622-34.

13. Visvanathan A, Patil V, Arora A, Hegde AS, Arivazhagan A, Santosh V, et al. Essential role of METTL3mediated $\mathrm{m}(6) \mathrm{A}$ modification in glioma stem-like cells maintenance and radioresistance. Oncogene. 2018;37(4):522-33.

14. Vu LP, Pickering BF, Cheng Y, Zaccara S, Nguyen D, Minuesa G, et al. The N(6)-methyladenosine $(m(6) A)$-forming enzyme METTL3 controls myeloid differentiation of normal hematopoietic and leukemia cells. Nat Med. 2017;23(11):1369-76.

15. Barbieri I, Tzelepis K, Pandolfini L, Shi J, Millan-Zambrano G, Robson SC, et al. Promoter-bound METTL3 maintains myeloid leukaemia by m(6)A-dependent translation control. Nature. 2017;552(7683):126-31.

16. Yue B, Song C, Yang L, Cui R, Cheng X, Zhang Z, et al. METTL3-mediated N6-methyladenosine modification is critical for epithelial-mesenchymal transition and metastasis of gastric cancer. Mol Cancer. 2019;18(1):142.

17. Zhang $C$, Chen $Y$, Sun B, Wang L, Yang Y, Ma D, et al. m(6)A modulates haematopoietic stem and progenitor cell specification. Nature. 2017;549(7671):273-6.

18. Lence T, Akhtar J, Bayer M, Schmid K, Spindler L, Ho CH, et al. m(6)A modulates neuronal functions and sex determination in Drosophila. Nature. 2016;540(7632):242-7.

19. Yu JM, Sun W, Wang ZH, Liang X, Hua F, Li K, et al. TRIB3 supports breast cancer stemness by suppressing FOXO1 degradation and enhancing SOX2 transcription. Nat Commun. 2019;10(1):5720.

20. Patterson KI, Brummer T, O'Brien PM, Daly RJ. Dual-specificity phosphatases: critical regulators with diverse cellular targets. Biochem J. 2009;418(3):475-89.

21. Meyer KD, Patil DP, Zhou J, Zinoviev A, Skabkin MA, Elemento O, et al. 5' UTR m(6)A Promotes CapIndependent Translation. Cell. 2015;163(4):999-1010.

22. Wang X, Zhao BS, Roundtree IA, Lu Z, Han D, Ma H, et al. N(6)-methyladenosine Modulates Messenger RNA Translation Efficiency. Cell. 2015;161(6):1388-99.

23. Lin S, Choe J, Du P, Triboulet R, Gregory RI. The m(6)A Methyltransferase METTL3 Promotes Translation in Human Cancer Cells. Mol Cell. 2016;62(3):335-45. 
24. Zhang S, Zhao BS, Zhou A, Lin K, Zheng S, Lu Z, et al. m(6)A Demethylase ALKBH5 Maintains Tumorigenicity of Glioblastoma Stem-like Cells by Sustaining FOXM1 Expression and Cell Proliferation Program. Cancer Cell. 2017;31(4):591-606 e6.

25. Lan T, Li H, Zhang D, Xu L, Liu H, Hao X, et al. KIAA1429 contributes to liver cancer progression through N6-methyladenosine-dependent post-transcriptional modification of GATA3. Mol Cancer. 2019;18(1):186.

26. Hanahan D, Weinberg RA. Hallmarks of cancer: the next generation. Cell. 2011;144(5):646-74.

27. Bolger AM, Lohse M, Usadel B. Trimmomatic: a flexible trimmer for Illumina sequence data. Bioinformatics. 2014;30(15):2114-20.

28. Langmead B, Trapnell C, Pop M, Salzberg SL. Ultrafast and memory-efficient alignment of short DNA sequences to the human genome. Genome Biol. 2009;10(3):R25.

29. Zhang Y, Liu T, Meyer CA, Eeckhoute J, Johnson DS, Bernstein BE, et al. Model-based analysis of ChIP-Seq (MACS). Genome Biol. 2008;9(9):R137.

30. Kolodziej-Wojnar P, Borkowska J, Wicik Z, Domaszewska-Szostek A, Polosak J, Cakala-Jakimowicz $\mathrm{M}$, et al. Alterations in the Genomic Distribution of $5 \mathrm{hmC}$ in In Vivo Aged Human Skin Fibroblasts. Int J Mol Sci. 2020;22(1).

31. Heinz S, Benner C, Spann N, Bertolino E, Lin YC, Laslo P, et al. Simple combinations of lineagedetermining transcription factors prime cis-regulatory elements required for macrophage and $\mathrm{B}$ cell identities. Mol Cell. 2010;38(4):576-89.

32. Kim D, Langmead B, Salzberg SL. HISAT: a fast spliced aligner with low memory requirements. Nat Methods. 2015;12(4):357-60.

33. Szklarczyk D, Morris JH, Cook H, Kuhn M, Wyder S, Simonovic M, et al. The STRING database in 2017: quality-controlled protein-protein association networks, made broadly accessible. Nucleic Acids Res. 2017;45(D1):D362-D8.

\section{Figures}


Figure 1

A

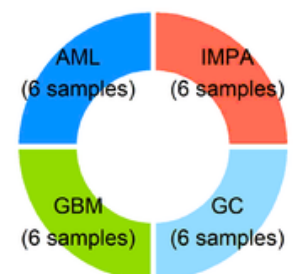

B

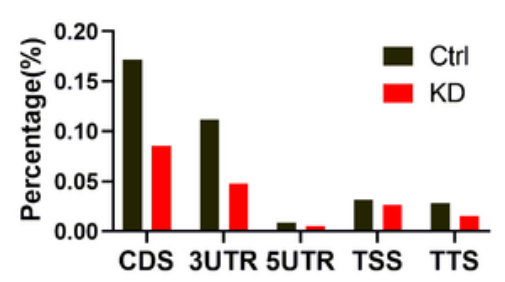

GC

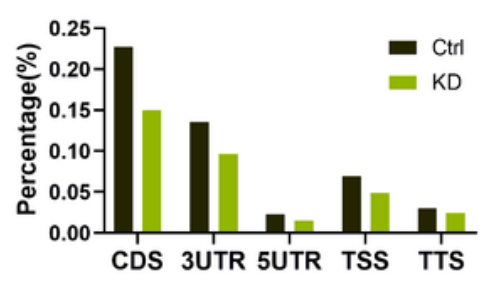

C
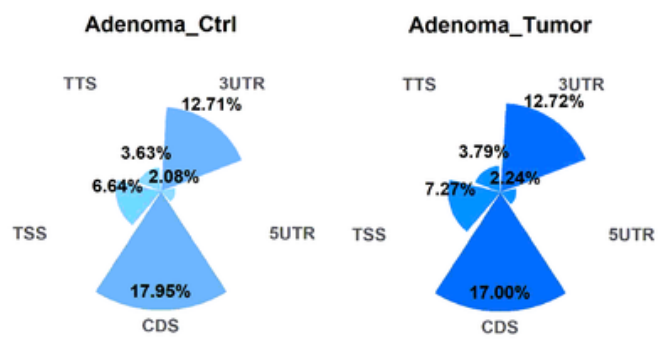

D

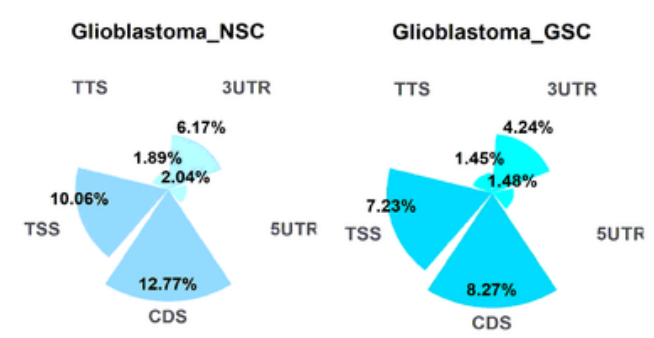

Adenoma

E

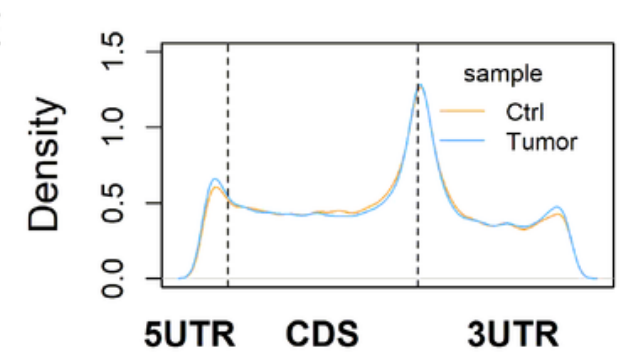

Gastric Cancer

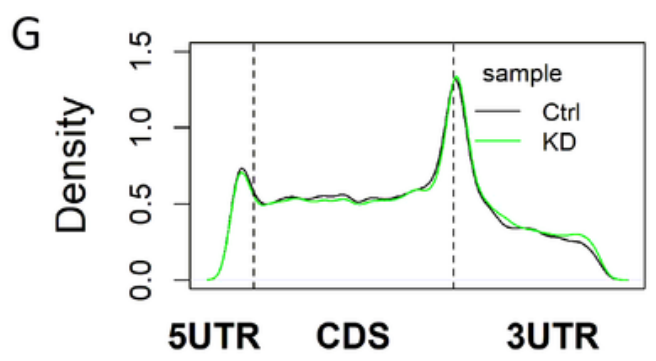

F

Myeloid Leukemia

F

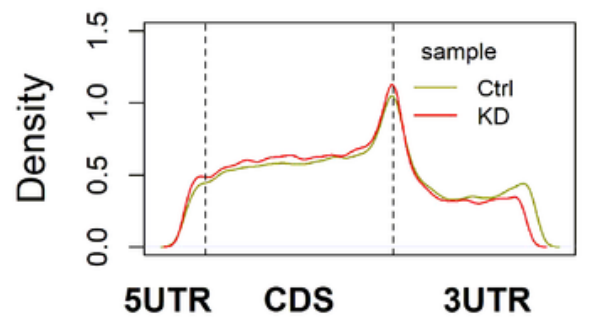

Glioblastoma

$\mathrm{H}$

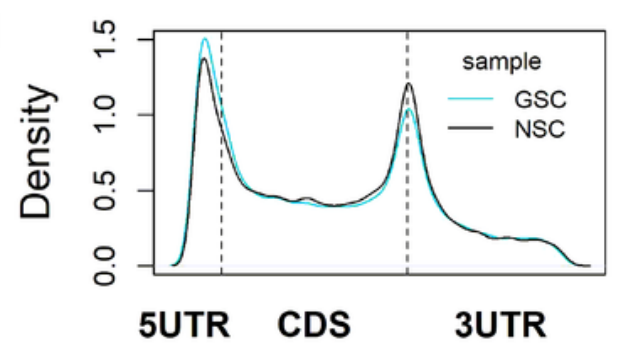

Figure 1

Distribution characteristics of m6A modification. Public data from the GEO database for analysis (A). Distribution of m6A enrichment peaks in different regions of the transcript in ML, GC, IMPA and GBM groups $(B, C, D)$. Peak density of m6A in different tumor samples $(E, F, G, H)$. 
Figure 2

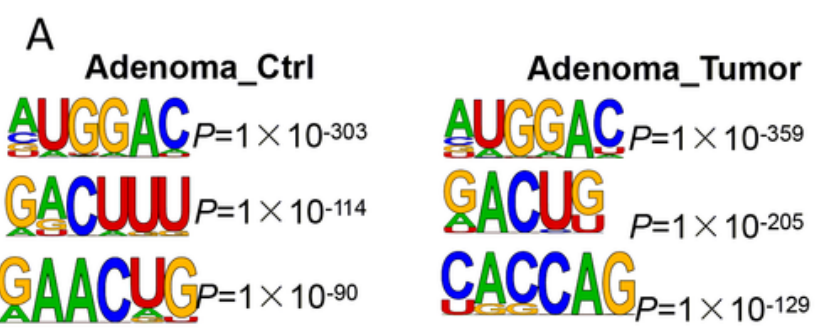

Glioblastoma_NSC

AUCGAC $P=1 \times 10^{-416}$

CAACAG $P=1 \times 10^{-109}$

AAACCU $P=1 \times 10^{-79}$

A C

C

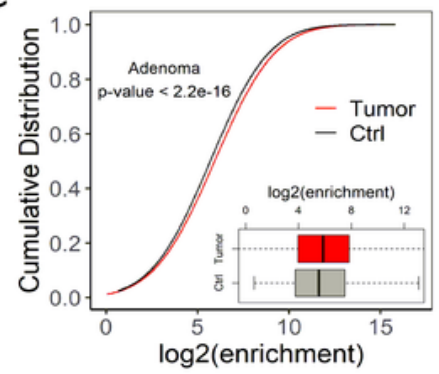

F

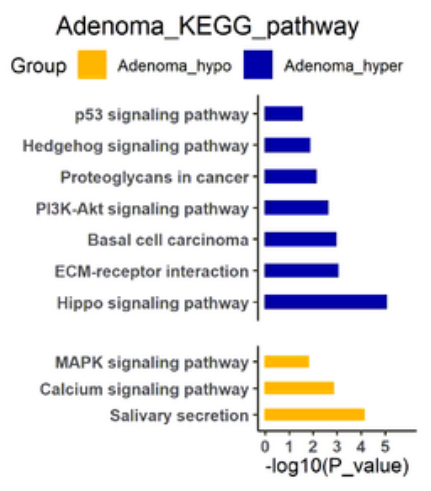

Glioblastoma_GSC SUGGAC $P=1 \times 10^{-258}$ AGACUU $P=1 \times 10.82$ UACUGU $P=1 \times 10^{-66}$ CCACAG $P=1 \times 10^{-36}$
G
B

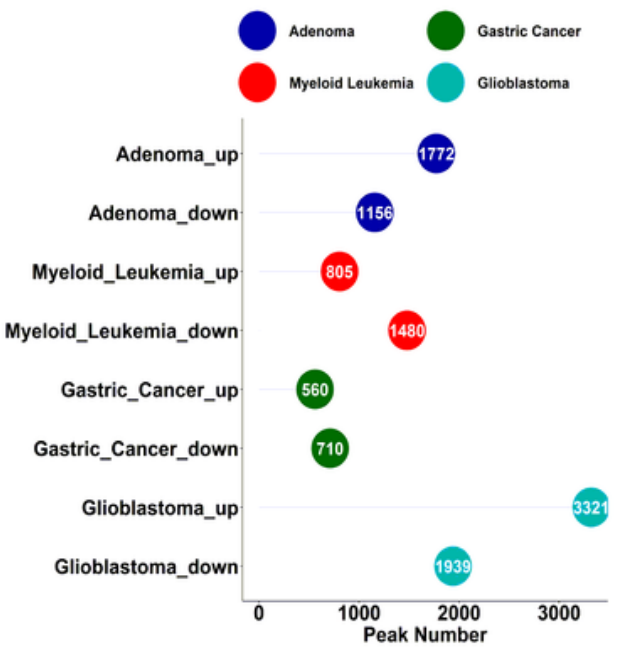

E
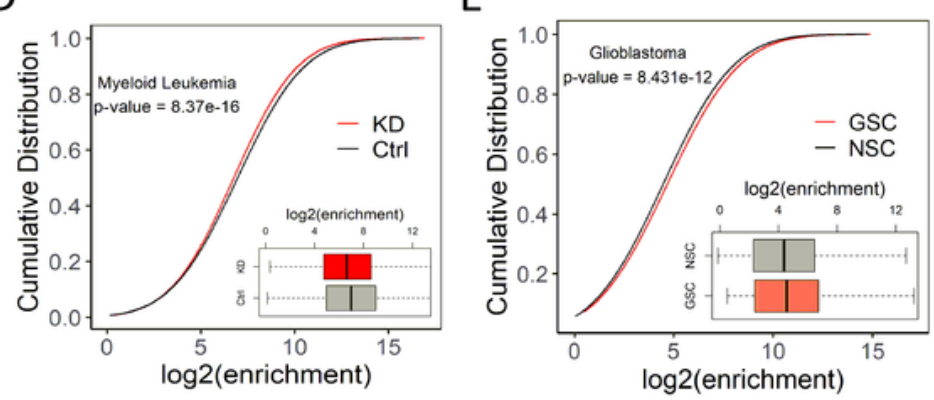

$\mathrm{H}$

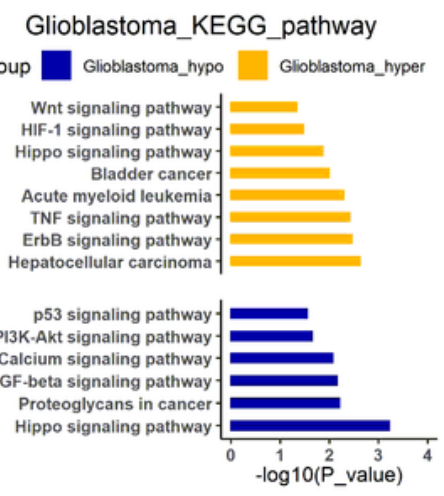

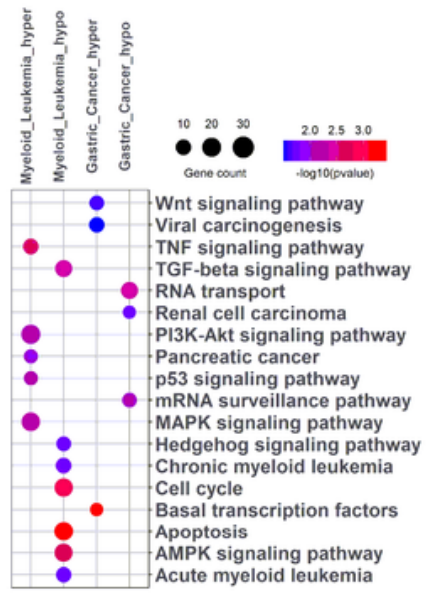

Figure 2

Comparison of m6A enrichment levels in different tumor samples and KEGG enrichment analysis for differential enrichment peak associated genes The m6A peaks in these tumor samples were characterized by the canonical motif $(A)$. The number of differential peaks identified in different tumor samples (B). Cumulative distribution curves of m6A enrichment in tumor samples compared to controls 
$(C, D, E)$. KEGG pathway enrichment analysis of genes associated with differential enrichment peaks (F, G, $\mathrm{H})$.

\section{Figure 3}

A

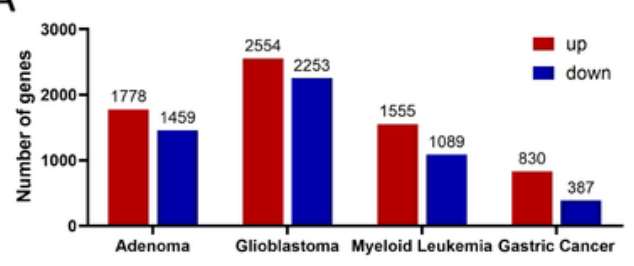

C
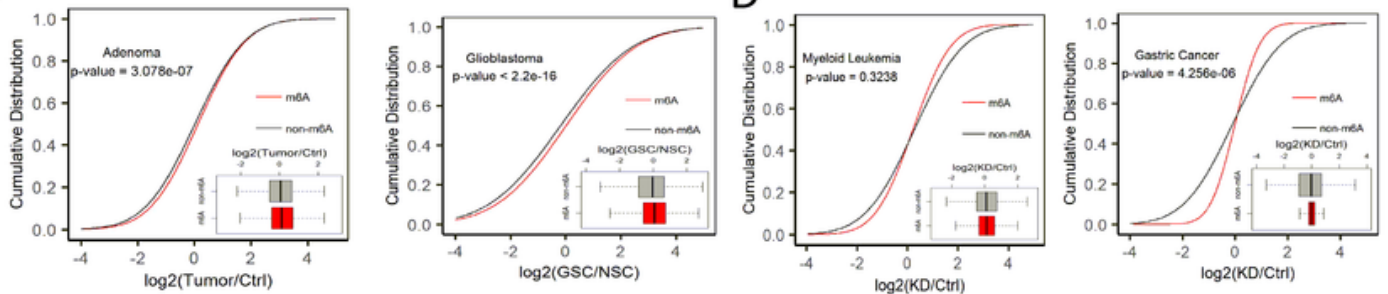

$\mathrm{E}$

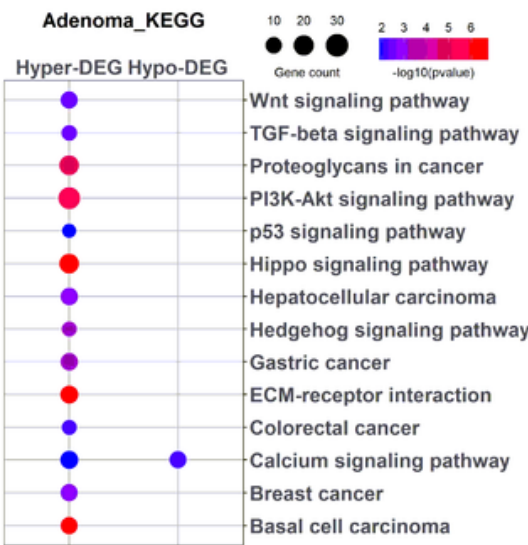

Myeloid Leukemia

G
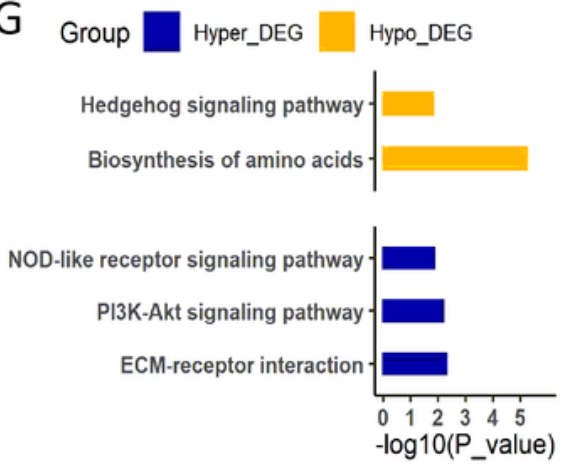
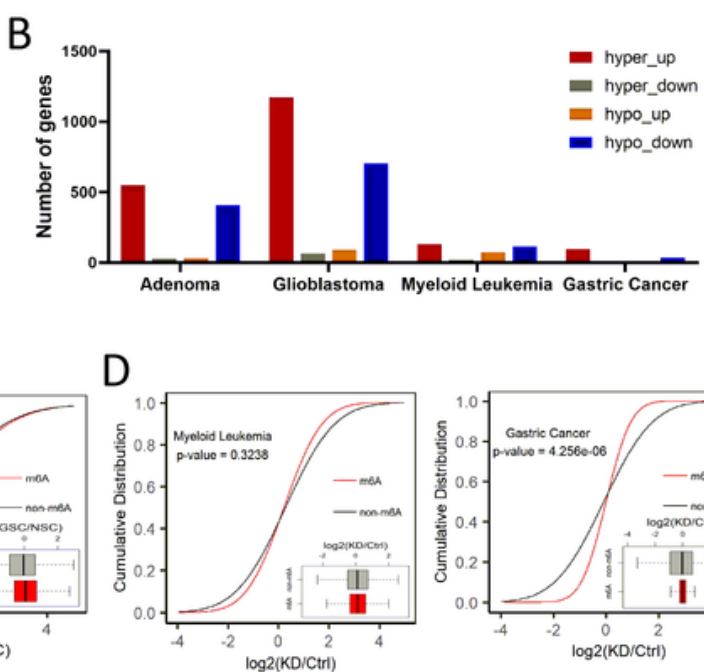

D

$\mathrm{F}$

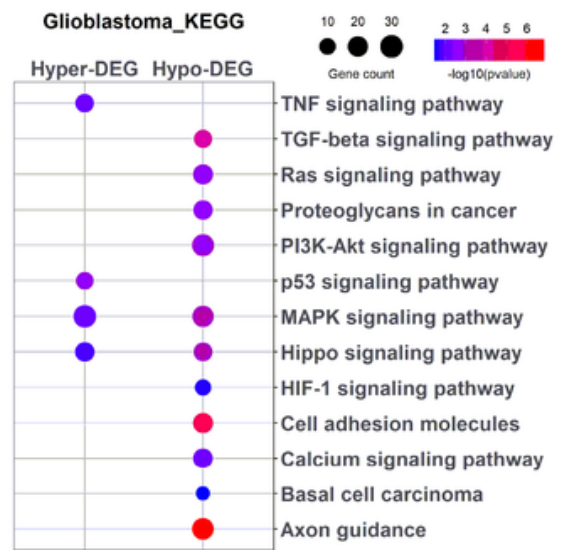

Gastric Cancer
Group $\square$ Hyper_DEG $\square$ Hypo_DEG
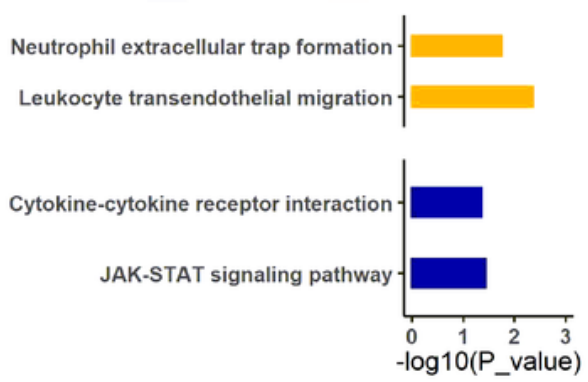

\section{Figure 3}

The role in genes expression by $\mathrm{m} 6 \mathrm{~A}$ enrichment peaks with dysregulation. Number of differentially expressed genes in different tumor groups (A). Differentially expressed genes related with hypermethylated and hypo-methylated peaks (B). Cumulative distribution curves of foldchanges (log2) 
between genes with m6A and non-m6A. The distributions were compared using Wilcoxon rank sum test (C, D). KEGG enrichment analysis for hyper-methylated peaks associated differentially expressed genes (Hyper-DEG) and hypo-methylated peaks associated differentially expressed genes (Hypo-DEG) in IMPA $(E), G B M(F), A M L(G)$ and $G C(H)$.

Figure 4

A

DEG

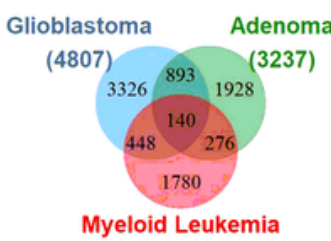

(2644)

C

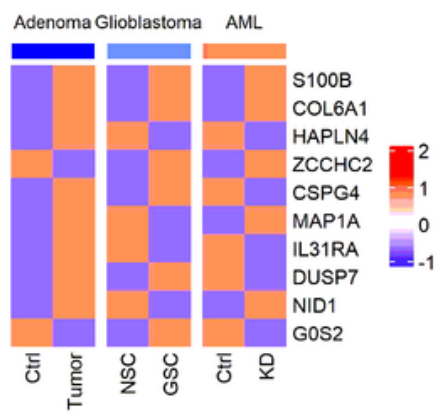

DMG

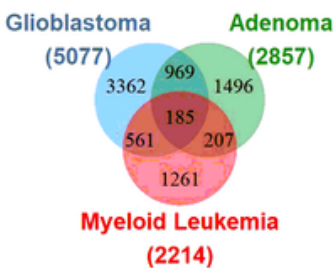

B

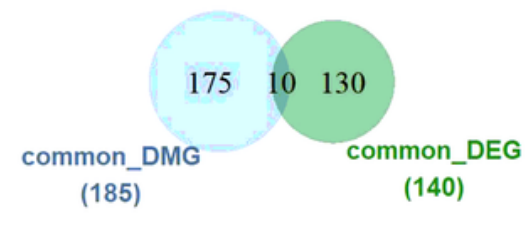

D
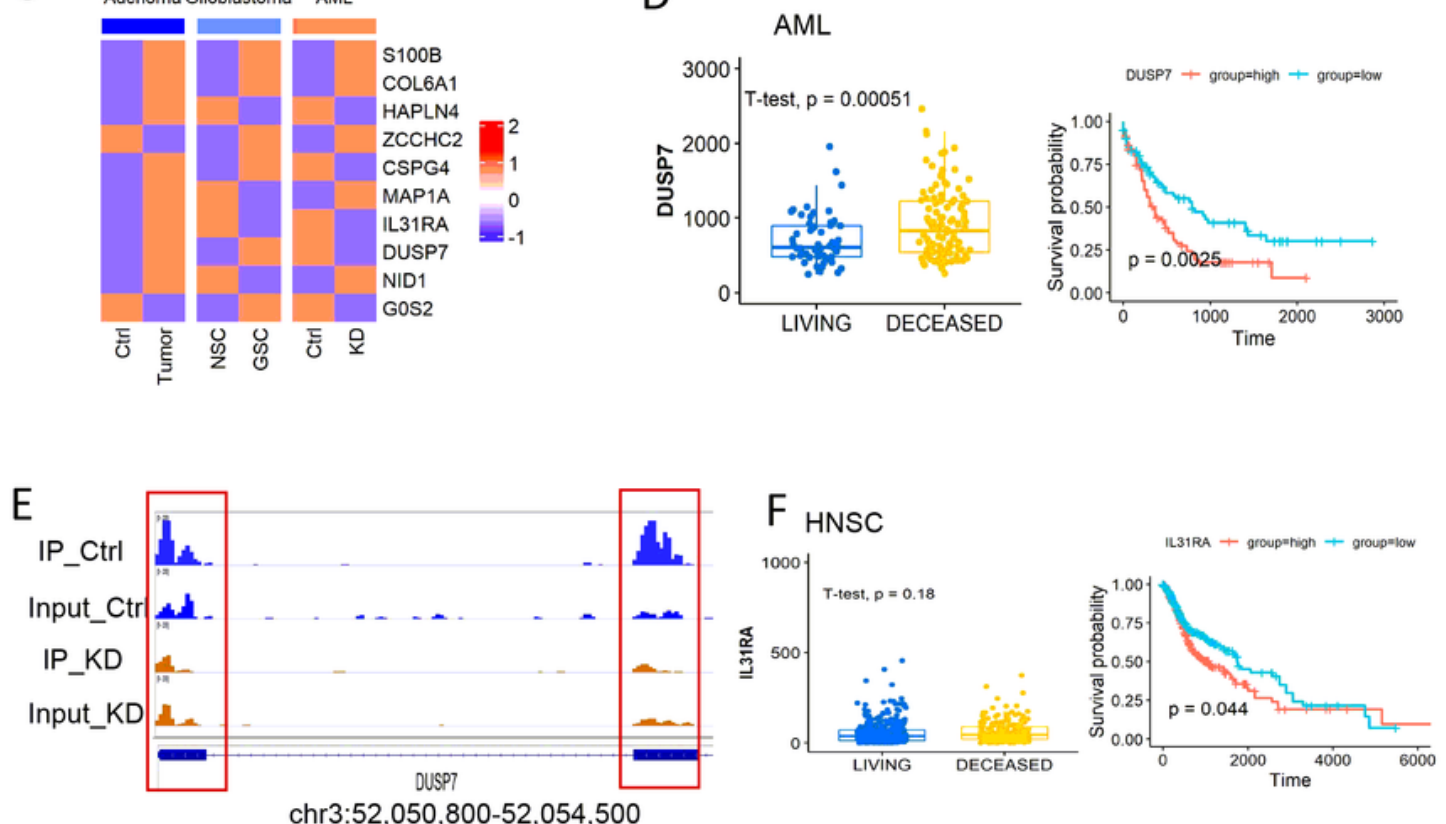

chr3:52,050,800-52,054,500

G
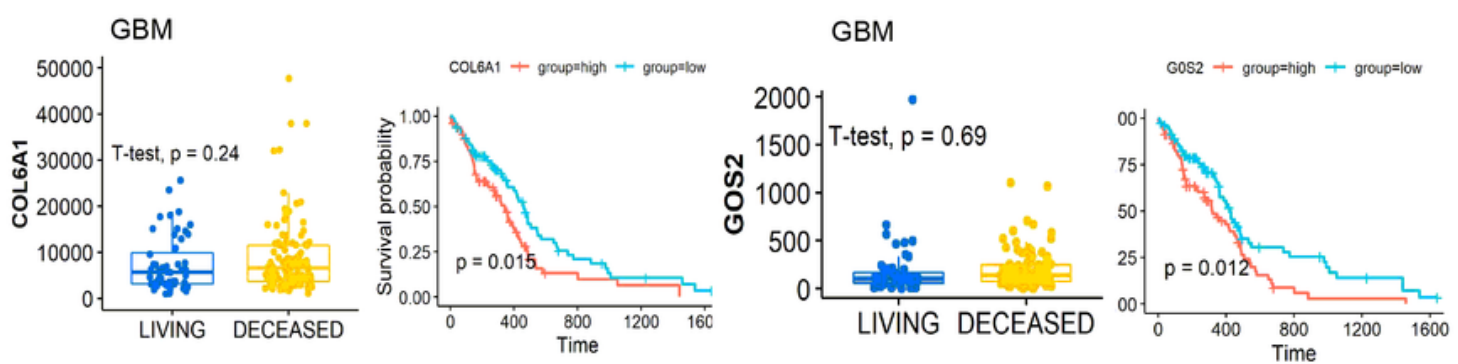

\section{Figure 4}


Effects of DEGs associated with differential m6A peaks on patients' overall survival Common differentially expressed genes (DEGs) and differential m6A methylation associated genes (DMGs) (A). There are 10 genes that are common between DEGs and DMGs (B). Expression patterns of 10 genes in tumor groups (C). High DUSP7 expression was significantly associated with poor overall survival in AML patients (D) and m6A modification enrichment of DUSP7 decreased after METLL3 knockdown (E). Effects of high expression of other genes on overall survival in patients with $\operatorname{HNSC}$ and $\operatorname{GBM}(F, G)$. Kaplan-Meier analysis was applied for survival analysis and differences between groups were analyzed using the log-rank test. 


\section{Figure 5}

A
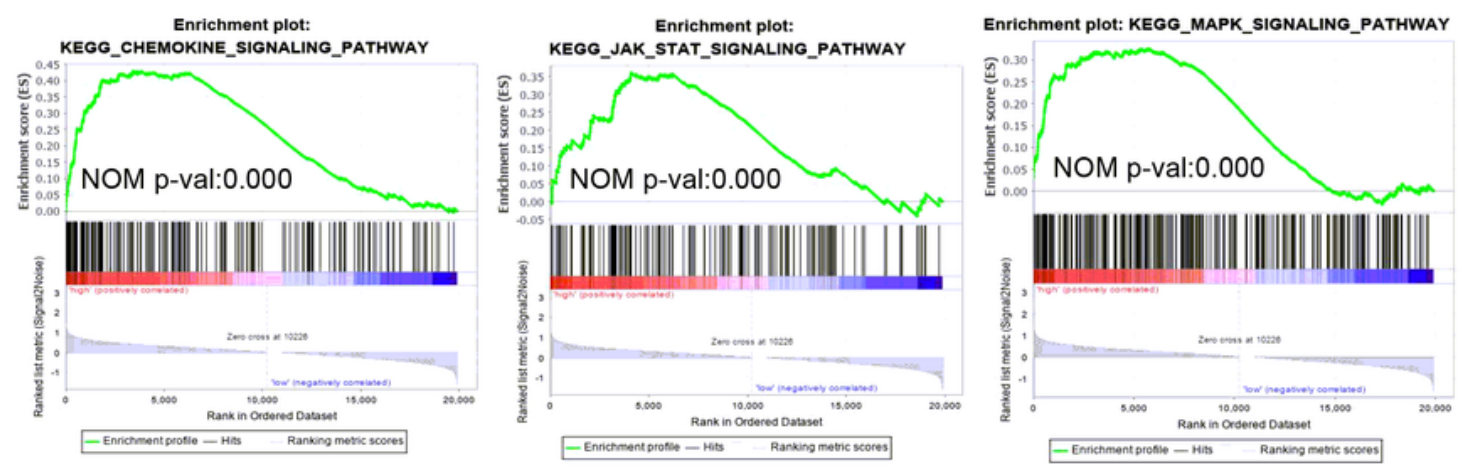

B
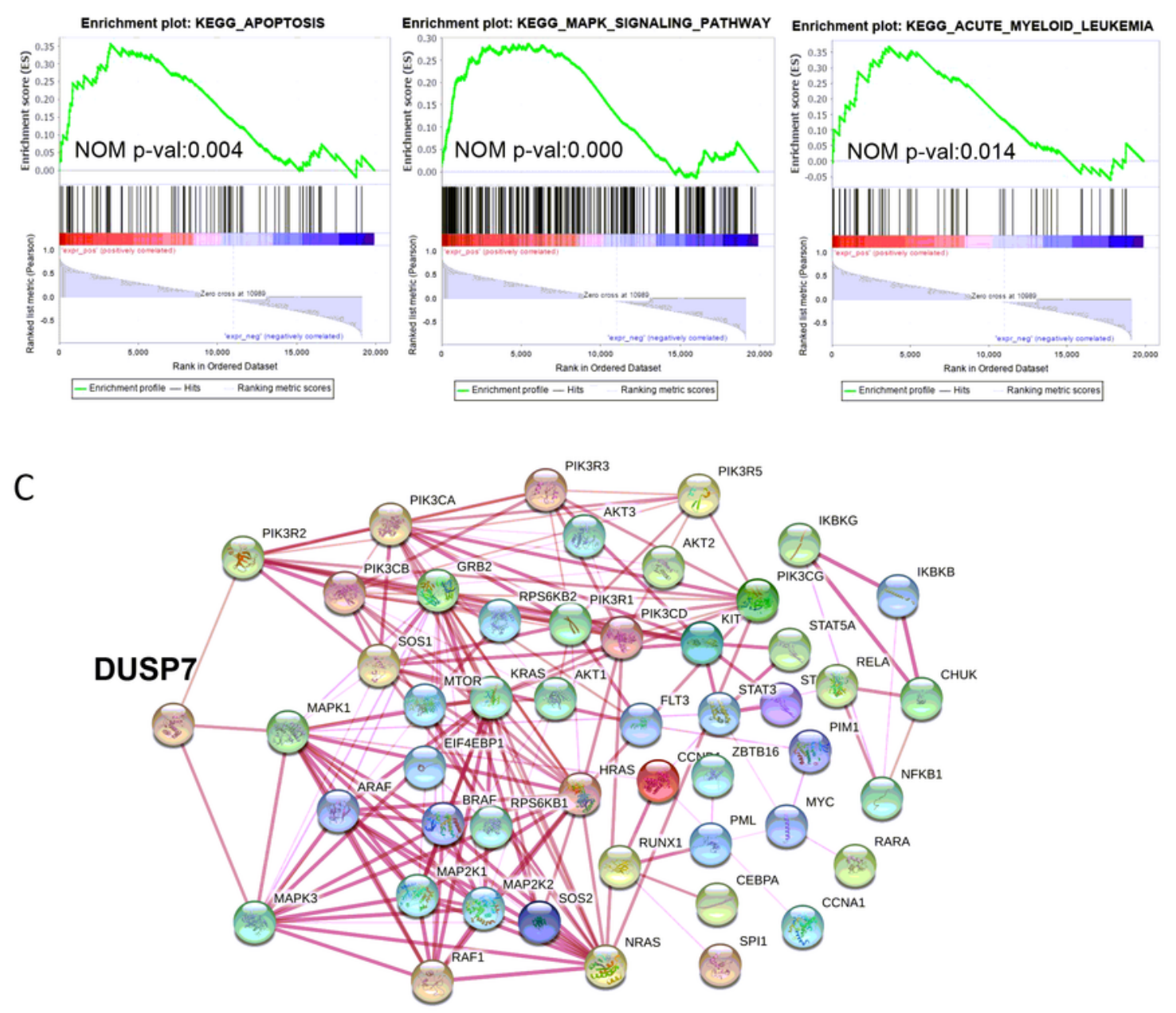

\section{Figure 5}

GSEA and protein interactions prove that MAPK signaling pathway is activated in DUSP7High and DUSP7Pos groups. KEGG pathways that are activated in the DUSP7High (A) and DUSP7Pos groups (B). Protein interaction results showed that DUSP7 was involved in AML progression through MAPKs and PIK3R2 proteins (C). 


\section{Supplementary Files}

This is a list of supplementary files associated with this preprint. Click to download.

- SupplementaryFigure1quwz.tif

- SupplementaryFigure2quwz.tif

- SupplementaryTable1bloodfilterdiffpeakDEGGOenrich.xIsx 